\title{
H.E.S.S. observations of the binary system PSR B1259-63/LS 2883 around the 2010/2011 periastron passage
}

H.E.S.S. Collaboration, A. Abramowski ${ }^{1}$, F. Acero ${ }^{2}$, F. Aharonian $3,4,5$, A. G. Akhperjanian ${ }^{6,5}$, G. Anton $^{7}$, S. Balenderan ${ }^{8}$, A. Balzer9,10, A. Barnacka ${ }^{11,12}$, Y. Becherini ${ }^{13,14,15}$, J. Becker Tjus ${ }^{16}$, K. Bernlöhr ${ }^{3,17}$, E. Birsin ${ }^{17}$, J. Biteau ${ }^{15}$, C. Boisson ${ }^{18}$, J. Bolmont ${ }^{19}$, P. Bordas ${ }^{20}$, J. Brucker ${ }^{7}$, F. Brun ${ }^{15}$, P. Brun ${ }^{12}$, T. Bulik ${ }^{21}$, S. Carrigan ${ }^{3}$, S. Casanova ${ }^{22,3}$, M. Cerruti ${ }^{18}$, P. M. Chadwick ${ }^{8}$, R. C. G. Chaves ${ }^{12,3}$, A. Cheesebrough ${ }^{8}$, S. Colafrancesco ${ }^{23}$, G. Cologna ${ }^{13}$, J. Conrad ${ }^{24}$, C. Couturier ${ }^{19}$, M. Dalton ${ }^{17,25,26}$, M. K. Daniel ${ }^{8}$, I. D. Davids ${ }^{27}$, B. Degrange ${ }^{15}$, C. Deil ${ }^{3}$, P. deWilt ${ }^{28}$, H. J. Dickinson ${ }^{24}$, A. Djannati-Atai ${ }^{14}$, W. Domainko ${ }^{3}$, L. O'C. Drury ${ }^{4}$, G. Dubus ${ }^{29}$, K. Dutson ${ }^{30}$, J. Dyks ${ }^{11}$, M. Dyrda ${ }^{31}$, K. Egberts ${ }^{32}$, P. Eger ${ }^{7}$, P. Espigat ${ }^{14}$, L. Fallon ${ }^{4}$, C. Farnier ${ }^{24}$, S. Fegan ${ }^{15}$, F. Feinstein ${ }^{2}$, M. V. Fernandes ${ }^{1}$, D. Fernandez ${ }^{2}$, A. Fiasson ${ }^{33}$, G. Fontaine ${ }^{15}$, A. Förster ${ }^{3}$, M. Füßling ${ }^{17}$, M. Gajdus ${ }^{17}$, Y. A. Gallant ${ }^{2}$, T. Garrigoux ${ }^{19}$, H. Gast ${ }^{3}$, B. Giebels ${ }^{15}$, J. F. Glicenstein ${ }^{12}$, B. Glück ${ }^{7}$, D. Göring ${ }^{7}$, M.-H. Grondin ${ }^{3,13}$, M. Grudzińska ${ }^{21}$, S. Häffner ${ }^{7}$, J. D. Hague ${ }^{3}$, J. Hahn ${ }^{3}$, D. Hampf ${ }^{1}$, J. Harris ${ }^{8}$, S. Heinz ${ }^{7}$, G. Heinzelmann ${ }^{1}$, G. Henri' ${ }^{29}$, G. Hermann ${ }^{3}$, A. Hillert ${ }^{3}$, J. A. Hinton ${ }^{30}$, W. Hofmann ${ }^{3}$, P. Hofverberg ${ }^{3}$, M. Holler ${ }^{10}$, D. Horns ${ }^{1}$, A. Jacholkowska ${ }^{19}$, C. Jahn ${ }^{7}$, M. Jamrozy ${ }^{34}$, I. Jung ${ }^{7}$, M. A. Kastendieck ${ }^{1}$, K. Katarzyński ${ }^{35}$, U. Katz ${ }^{7}$, S. Kaufmann ${ }^{13}$, B. Khélifi ${ }^{15}$, S. Klepser ${ }^{9}$, D. Klochkov ${ }^{20}$, W. Kluźniak ${ }^{11}$, T. Kneiske ${ }^{1}$, D. Kolitzus ${ }^{32}$, Nu. Komin ${ }^{33}$, K. Kosack ${ }^{12}$, R. Kossakowski ${ }^{33}$, F. Krayzel ${ }^{33}$, P. P. Krüger ${ }^{22,3}$, H. Laffon ${ }^{25,15}$, G. Lamanna ${ }^{33}$, J. Lefaucheur ${ }^{14}$, M. Lemoine-Goumard ${ }^{25}$, J.-P. Lenain ${ }^{19}$, D. Lennarz ${ }^{3}$,

T. Lohse ${ }^{17}$, A. Lopatin ${ }^{7}$, C.-C. Lu $^{3}$, V. Marandon ${ }^{3}$, A. Marcowith ${ }^{2}$, J. Masbou ${ }^{33}$, G. Maurin ${ }^{33}$, N. Maxted ${ }^{28}$, M. Mayer ${ }^{10}$, T. J. L. McComb ${ }^{8}$, M. C. Medina ${ }^{12}$, J. Méhault ${ }^{2,25,26}$, U. Menzler ${ }^{16}$, R. Moderski ${ }^{11}$, M. Mohamed ${ }^{13}$, E. Moulin ${ }^{12}$, C. L. Naumann ${ }^{19}$, M. Naumann-Godo ${ }^{12}$, M. de Naurois ${ }^{15, \star}$, D. Nedbal ${ }^{36}$, N. Nguyen ${ }^{1}$, J. Niemiec $^{31}$, S. J. Nolan ${ }^{8}$, L. Oakes ${ }^{17}$, S. Ohm ${ }^{30,37}$, E. de Oña Wilhelmi ${ }^{3}$, B. Opitz ${ }^{1}$, M. Ostrowski ${ }^{34}$, I. Oya ${ }^{17}$, M. Panter ${ }^{3}$, R. D. Parsons ${ }^{3}$, M. Paz Arribas ${ }^{17}$, N. W. Pekeur ${ }^{22}$, G. Pelletier ${ }^{29}$, J. Perez ${ }^{32}$, P.-O. Petrucci ${ }^{29}$, B. Peyaud ${ }^{12}$, S. Pita ${ }^{14}$,

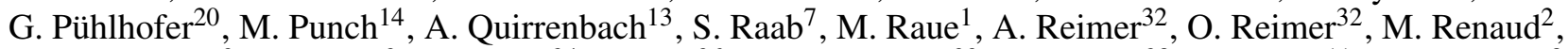
R. de los Reyes ${ }^{3}$, F. Rieger ${ }^{3}$, J. Ripken ${ }^{24}$, L. Rob ${ }^{36}$, S. Rosier-Lees ${ }^{33}$, G. Rowell ${ }^{28}$, B. Rudak ${ }^{11}$, C. B. Rulten ${ }^{8}$, V. Sahakian ${ }^{6,5}$, D. A. Sanchez ${ }^{3}$, A. Santangelo ${ }^{20}$, R. Schlickeiser ${ }^{16}$, A. Schulz ${ }^{9}$, U. Schwanke ${ }^{17}$, S. Schwarzburg ${ }^{20}$, S. Schwemmer ${ }^{13}$, F. Sheidaei ${ }^{14,22}$, J. L. Skilton ${ }^{3}$, H. Sol ${ }^{18}$, G. Spengler ${ }^{17}$, Ł. Stawarz ${ }^{34}$, R. Steenkamp ${ }^{27}$, C. Stegmann ${ }^{10,9}$, F. Stinzing ${ }^{7}$, K. Stycz ${ }^{9}$, I. Sushch ${ }^{17, \star}$, A. Szostek ${ }^{34}$, J.-P. Tavernet ${ }^{19}$, R. Terrier ${ }^{14}$, M. Tluczykont ${ }^{1}$, C. Trichard ${ }^{33}$, K. Valeriuss ${ }^{7}$, C. van Eldik ${ }^{7,3}$, G. Vasileiadis ${ }^{2}$, C. Venter ${ }^{22}$, A. Viana ${ }^{12,3}$, P. Vincent ${ }^{19}$, H. J. Völk ${ }^{3}$,

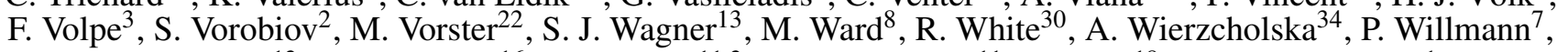
D. Wouters ${ }^{12}$, M. Zacharias ${ }^{16}$, A. Zajczyk ${ }^{11,2}$, A. A. Zdziarski ${ }^{11}$, A. Zech ${ }^{18}$, and H.-S. Zechlin ${ }^{1}$

\section{(Affiliations can be found after the references)}

Received 22 October 2012 / Accepted 8 January 2013

\section{ABSTRACT}

Aims. We present very high energy (VHE; $E>100 \mathrm{GeV}$ ) data from the $\gamma$-ray binary system PSR B1259-63/LS 2883 taken around its periastron passage on 15th of December 2010 with the High Energy Stereoscopic System (H.E.S.S.) of Cherenkov Telescopes. We aim to search for a possible $\mathrm{TeV}$ counterpart of the GeV flare detected by the Fermi LAT. In addition, we aim to study the current periastron passage in the context of previous observations taken at similar orbital phases, testing the repetitive behaviour of the source.

Methods. Observations at VHEs were conducted with H.E.S.S. from 9th to 16 th of January 2011 . The total dataset amounts to $\sim 6 \mathrm{~h}$ of observing time. The data taken around the 2004 periastron passage were also re-analysed with the current analysis techniques in order to extend the energy spectrum above $3 \mathrm{TeV}$ to fully compare observation results from 2004 and 2011.

Results. The source is detected in the 2011 data at a significance level of $11.5 \sigma$ revealing an averaged integral flux above $1 \mathrm{TeV}$ of $(1.01 \pm$ $\left.0.18_{\text {stat }} \pm 0.20_{\text {sys }}\right) \times 10^{-12} \mathrm{~cm}^{-2} \mathrm{~s}^{-1}$. The differential energy spectrum follows a power-law shape with a spectral index $\Gamma=2.92 \pm 0.30_{\text {stat }} \pm 0.20_{\text {sys }}$ and a flux normalisation at $1 \mathrm{TeV}$ of $N_{0}=\left(1.95 \pm 0.32_{\text {stat }} \pm 0.39_{\text {sys }}\right) \times 10^{-12} \mathrm{TeV}^{-1} \mathrm{~cm}^{-2} \mathrm{~s}^{-1}$. The measured light curve does not show any evidence for variability of the source on the daily scale. The re-analysis of the 2004 data yields results compatible with the published ones. The differential energy spectrum measured up to $\sim 10 \mathrm{TeV}$ is consistent with a power law with a spectral index $\Gamma=2.81 \pm 0.10_{\text {stat }} \pm 0.20_{\text {sys }}$ and a flux normalisation at $1 \mathrm{TeV}$ of $N_{0}=\left(1.29 \pm 0.08_{\text {stat }} \pm 0.26_{\text {sys }}\right) \times 10^{-12} \mathrm{TeV}^{-1} \mathrm{~cm}^{-2} \mathrm{~s}^{-1}$.

Conclusions. The measured integral flux and the spectral shape of the 2011 data are compatible with the results obtained around previous periastron passages. The absence of variability in the H.E.S.S. data indicates that the GeV flare observed by Fermi LAT in the time period covered also by H.E.S.S. observations originates in a different physical scenario than the $\mathrm{TeV}$ emission. Moreover, the comparison of the new results to the results from the 2004 observations made at a similar orbital phase provides a stronger evidence of the repetitive behaviour of the source.

Key words. gamma rays: general - pulsars: individual: PSR B1259-63 - X-rays: binaries - stars: individual: LS 2883

* Corresponding authors: e-mail: yusushch@physik.hu-berlin.de; denauroi@in2p3.fr 


\section{Introduction}

The class of very high energy (VHE; $E>100 \mathrm{GeV}) \gamma$-ray binaries comprises only a handful of known objects in our Galaxy: LS 5039 (Aharonian et al. 2005a), LS I +61 303 (Albert et al. 2006), PSR B1259-63/LS 2883 (Aharonian et al. 2005b) and HESS J0632+057 (Aharonian et al. 2007), the first binary primarily discovered at $\mathrm{TeV}$ energies. This class can be extended by two more candidates: Cygnus X-1 (Albert et al. 2007), a stellarmass black hole binary detected at VHEs at the $4.1 \sigma$ significance level, and HESS J1018-589 (HESS Collaboration et al. 2012), which shows a point-like component spatially coincident with the GeV binary 1FGL J1018.6-5856 discovered by the Fermi LAT collaboration (Abdo et al. 2010). Only for the source presented in this paper, PSR B1259-63/LS 2883, the compact companion is clearly identified as a pulsar, making it a unique object for the study of the interaction between pulsar and stellar winds and the emission mechanisms in such systems.

PSR B1259-63/LS 2883 was discovered in a high-frequency radio survey devoted to the detection of young, distant, and short-period pulsars (Johnston et al. 1992a,b). It consists of a rapidly rotating pulsar with a spin period of $\simeq 48 \mathrm{~ms}$ and a spindown luminosity of $\simeq 8 \times 10^{35} \mathrm{erg} / \mathrm{s}$ in a highly eccentric $(e=$ 0.87 ) orbit around a massive Be star. The pulsar moves around the companion with a period $P_{\text {orb }}=3.4$ years $(1237$ days $)$.

Latest optical observations with VLT UT2 (Negueruela et al. 2011) significantly improved the previously known parameters of the companion star LS 2883. The luminosity of the star is $L_{*}=2.3 \times 10^{38} \mathrm{erg} \mathrm{s}^{-1}$. Because of its fast rotation, the star is oblate with an equatorial radius of $R_{\text {eq }}=9.7 R_{\odot}$ and a polar radius of $R_{\text {pole }}=8.1 R_{\odot}$. This leads to a strong gradient of the surface temperature from $T_{\mathrm{eq}} \approx 27500 \mathrm{~K}$ at the equator to $T_{\text {pole }} \approx 34000 \mathrm{~K}$ at the poles. The mass function of the system suggests a mass of the star $M_{*} \approx 30 M_{\odot}$ and an orbital inclination angle $i_{\text {orb }} \approx 25^{\circ}$ for the smallest neutron star mass of $1.4 M_{\odot}$. The optical observations also suggest that the system is located at the same distance as the star association Cen OB1 at $d=(2.3 \pm 0.4) \mathrm{kpc}$ (Negueruela et al. 2011). The companion Be star features an equatorial disk that is believed to be inclined with respect to the pulsar's orbital plane (Johnston et al. 1992a; Melatos et al. 1995; Negueruela et al. 2011) in a way that the pulsar crosses the disk twice in each orbit just before ( $\sim 20$ days $)$ and just after ( 20 days) the periastron.

Since its discovery in 1992, PSR B1259-63/LS 2883 is constantly monitored by various instruments at all energy bands. The source shows broadband emission and is visible from radio wavelengths up to the VHE regime. The properties of the radio emission differ depending on the distance between the pulsar and the star. Radio observations (Johnston et al. 1999, 2005; Connors et al. 2002) show that when the pulsar is far from the periastron, the observed radio emission consists only of the pulsed component, whose intensity is almost independent of the orbital position. But closer to the periastron, starting at about $t_{\mathrm{p}}-100 \mathrm{~d}$, where $t_{\mathrm{p}}$ is the time of periastron, the intensity starts to decrease up to the complete disappearance approximately at $t_{\mathrm{p}}-20 \mathrm{~d}$. This is followed by an eclipse of the pulsed emission for about 35-40 days when the pulsar is behind the disk. In contrast, a transient unpulsed component appears and sharply rises to a level more than ten times higher than the flux density of the pulsed emission far from the periastron. The unpulsed component is believed to come from synchrotron radiation generated in the shocked wind zone between the relativistic pulsar wind and the stellar disk outflow. After the disk crossing the unpulsed emission shows a slight decrease with another increase around $t_{\mathrm{p}}+20 \mathrm{~d}$ at the second crossing of the disk. Radio observations around the 2007 periastron passage showed extended unpulsed emission with a total projected extent of $\sim 120 \mathrm{AU}$ and the peak of the emission clearly displaced from the binary system orbit (Moldón et al. 2011). This indicates that a flow of synchrotronemitting particles, which can travel far away from the system, can be produced in PSR B1259-63/LS 2883. The source was also monitored around the 2010 periastron passage. The pulsed radio emission was monitored with the Parkes telescope, revealing an eclipse of the pulsed signal that lasted from $t_{\mathrm{p}}-16 \mathrm{~d}$ to $t_{\mathrm{p}}+15 \mathrm{~d}$. Radio emission from PSR B1259-63 at frequencies between 1.1 and $10 \mathrm{GHz}$ was observed using the ATCA array in the period from $t_{\mathrm{p}}-31 \mathrm{~d}$ to $t_{\mathrm{p}}+55 \mathrm{~d}$. The detected unpulsed emission around the periastron passage showed a behaviour similar to the one observed during previous observations (Abdo et al. 2011).

PSR B1259-63/LS 2883 is very well covered by X-ray observations carried out with various instruments such as ROSAT (Cominsky et al. 1994), ASCA (Kaspi et al. 1995; Hirayama et al. 1999), INTEGRAL (Shaw et al. 2004), and XMM-Newton (Chernyakova et al. 2006). The periastron passage in 2007 was monitored at the same time by Suzaku, Swift, XMM-Newton, and Chandra (Chernyakova et al. 2009). Observations around the 2010 periastron passage were performed by three instruments: Swift, Suzaku, and XMM-Newton (Abdo et al. 2011). Observations confirmed the $1-10 \mathrm{keV}$ light curve shape obtained in previous periastron observations, showing a rapid $\mathrm{X}$-ray brightening that started at about $t_{\mathrm{p}}-25 \mathrm{~d}$ with a subsequent decrease closer to periastron and a second increase of the X-ray flux after periastron (Abdo et al. 2011). X-ray observations did not show any X-ray pulsed emission from the pulsar. Unpulsed non-thermal radiation from the source appeared to be variable in flux and spectral index. Similarly to radio measurements, the enhancement of the flux occurs shortly before and shortly after the periastron. Unambiguously, the enhancement of the non-thermal emission results from the interaction of the pulsar wind with the circumstellar disk close to the periastron passage.

PSR B1259-63/LS 2883 was observed by H.E.S.S. around the periastron passages in 2004 (Aharonian et al. 2005b) and 2007 (Aharonian et al. 2009), leading to a firm detection on both occasions. In 2004, PSR B1259-63/LS 2883 was observed mostly after the periastron, in 2007 mostly before it. Therefore, the repetitive behaviour of the source, i.e. the recurrent appearance of the source near periastron at the same orbital phase, with the same flux level and spectral shape of the emission, was not precisely confirmed, since the observations covered different orbital phases. However, the similar dependence of the flux on the separation distance between the pulsar and the star for both periastron passages provides a strong indication for the repetitive behaviour (Kerschhaggl 2011). PSR B1259-63/LS 2883 was not detected in observations performed far from periastron in 2005 and 2006, which comprised $8.9 \mathrm{~h}$ and $7.5 \mathrm{~h}$ of exposure, respectively.

Observations around the 2004 and 2007 periastron passages showed a variable behaviour of the source flux with time. A combined light curve of those two periastron passages indicates two asymmetrical peaks around periastron with a significant decrease of the flux at the periastron itself. Peaks of the TeV emission roughly coincide with the flux enhancement observed in other wavebands as well as with the eclipse of the pulsed radio emission, which indicates the position of the circumstellar disk. This coincidence suggests that the TeV emission from PSR B1259-63/LS 2883 may be connected to the interaction of the pulsar with the disk. 
Table 1. Analysis results of the H.E.S.S. data for the full observation period as well as for the pre-flare and flare periods.

\begin{tabular}{l|ccccc|cccc}
\hline \hline Dataset & Livetime $[\mathrm{h}]$ & $N_{\mathrm{ON}}$ & $N_{\mathrm{OFF}}$ & $\alpha$ & $N_{\gamma}$ & Significance $[\sigma]$ & $\Gamma$ & $N_{0}\left[10^{-12} \mathrm{TeV}^{-1} \mathrm{~cm}^{-2} \mathrm{~s}^{-1}\right]$ & $\begin{array}{c}\text { Flux }(E>1 \mathrm{TeV}) \\
{\left[10^{-12} \mathrm{~cm}^{-2} \mathrm{~s}^{-1}\right]}\end{array}$ \\
\hline Full & 6.2 & 112 & 365 & 0.077 & 84.0 & 11.5 & $2.92 \pm 0.30_{\text {stat }} \pm 0.20_{\text {syst }}$ & $1.95 \pm 0.32_{\text {stat }} \pm 0.39_{\text {syst }}$ & $1.01 \pm 0.18_{\text {stat }} \pm 0.20_{\text {syst }}$ \\
Pre-flare & 2.65 & 44 & 133 & 0.076 & 33.9 & 7.4 & $2.94 \pm 0.52_{\text {stat }} \pm 0.20_{\text {syst }}$ & $2.15 \pm 0.56_{\text {stat }} \pm 0.43_{\text {syst }}$ & $1.11 \pm 0.29_{\text {stat }} \pm 0.22_{\text {syst }}$ \\
Flare & 3.59 & 68 & 232 & 0.077 & 50.1 & 8.5 & $3.26 \pm 0.49_{\text {stat }} \pm 0.20_{\text {syst }}$ & $1.81 \pm 0.39_{\text {stat }} \pm 0.36_{\text {syst }}$ & $0.80 \pm 0.22_{\text {stat }} \pm 0.16_{\text {syst }}$ \\
\hline
\end{tabular}

Notes. The pre-flare and flare periods are defined by the beginning of the HE flare (see text). $N_{\mathrm{ON}}$ and $N_{\mathrm{OFF}}$ are numbers of ON and OFF events, $\alpha$ is the background normalisation and $N_{\gamma}$ is the number of excess photon events.

The paper is organised as follows: in Sect. 2, Fermi-LAT observations at the 2010/2011 periastron passage are reviewed. In Sect. 3, the dataset and analysis techniques are described and analysis results are presented. Results are discussed in Sect. 4 and summarised in Sect. 5.

\section{Fermi-LAT detection of a post-periastron HE flare}

H.E.S.S. observations around the most recent periastron passage, which took place on 15th of December 2010, were performed as part of an extended multiwavelength (MWL) campaign, which included also radio, optical, X-ray and, for the first time, highenergy (HE; $E>100 \mathrm{MeV}$ ) observations. This paper is dedicated to the study of the H.E.S.S. results in the context of the HE observations. The detailed study of the MWL emission from the source will be presented in the joint MWL paper, which is currently in preparation.

Observations of the binary system PSR B1259-63/LS 2883 at HEs were performed using the Large Area Telescope (LAT) on board of Fermi. The data taken around the periastron passage were analysed by two independent working groups (Abdo et al. 2011; Tam et al. 2011), yielding similar results for the flaring period (see below), although there are some discrepancies related to the first detection period close to the periastron passage. Those differences do not affect the conclusions drawn in this paper, however. The source was detected close to periastron with a very low photon flux above $100 \mathrm{MeV}$ of about $(1-2) \times 10^{-7} \mathrm{~cm}^{-2} \mathrm{~s}^{-1}$. After the initial detection, the flux decreased and the source was below the detection threshold until 14th of January, $t_{\mathrm{p}}+30 \mathrm{~d}$, when a spectacular flare was detected, which lasted for about seven weeks with an average flux that was about 10 times higher than the flux detected close to periastron (Abdo et al. 2011). The highest day-averaged flux during the flare almost reached the estimate of the spin-down luminosity of the pulsar, which indicates a close to $100 \%$ efficiency of the conversion of the pulsar's rotational energy into $\gamma$-rays. The HE emission around the periastron as a function of time significantly differs from the two-peak light curves observed in other wavebands. The flare is not coincident with the post-periastron peak in radio, X-rays, and VHE $\gamma$-rays. It is also much brighter than the $\mathrm{GeV}$ emission detected close to the periastron passage (Abdo et al. 2011; Tam et al. 2011).

\section{H.E.S.S. observations and analysis}

\subsection{The H.E.S.S. instrument}

H.E.S.S. (High Energy Stereoscopic System) is an array of four $13 \mathrm{~m}$ diameter imaging atmospheric Cherenkov telescopes located in the Khomas Highland, Namibia, at an altitude of $1800 \mathrm{~m}$ above sea level (Hinton 2004). The telescopes are optimised for the detection of VHE $\gamma$-rays in the range from $100 \mathrm{GeV}$ to several tens of $\mathrm{TeV}$ by imaging Cherenkov light emitted by charged particles in extensive air showers. The total field of view of H.E.S.S. is $5^{\circ}$. The angular resolution of the system is $\lesssim 0.1^{\circ}$ and the average energy resolution is about $15 \%$. The H.E.S.S. array is capable of detecting point sources with a flux of $1 \%$ of the Crab nebula flux at a significance level of $5 \sigma$ in $25 \mathrm{~h}$ when observing at low zenith angles (Aharonian et al. 2006).

\subsection{Data set and analysis techniques}

PSR B1259-63/LS 2883 observations were scheduled to cover the post-periastron period from January to March 2011. The source was not visible for H.E.S.S. before and at the periastron passage. The observations resulted in a rather small dataset due to unfavourable weather conditions. The collected data correspond to $6 \mathrm{~h}$ of livetime after the standard quality selection procedure (Aharonian et al. 2006). These data were taken in five nights, namely January $9 / 10,10 / 11,13 / 14,14 / 15$ and $15 / 16$. The observations were performed at a relatively high average zenith angle of $48^{\circ}$ and with a mean offset angle of $0.55^{\circ}$ from the test region centred at $\alpha_{\mathrm{J} 2000}=13^{\mathrm{h}} 02^{\mathrm{m}} 48^{\mathrm{s}}, \delta_{\mathrm{J} 2000}=-63^{\circ} 50^{\prime} 09^{\prime \prime}$ (Wang et al. 2004).

The data were analysed using the model analysis ${ }^{1}$ technique with standard cuts (de Naurois \& Rolland 2009). The test region was a priori defined as a circle with radius $0.1^{\circ}$ (i.e. $\theta^{2}<0.01^{\mathrm{o}^{2}}$, where $\theta$ is defined as the angular distance between the $\gamma$-ray event and the nominal target position), which is the standard size for point-like sources. The reflected region background technique was used for the background subtraction. The analysis results were cross-checked with an alternative analysis chain ${ }^{2}$ using a standard Hillas reconstruction (Aharonian et al. 2006) method for $\gamma$ /hadron separation and an independent calibration of the raw data. Both analysis chains yielded consistent results.

\subsection{Energy spectrum}

The source was detected at an $11.5 \sigma$ level ( $\mathrm{Li} \& \mathrm{Ma} 1983$ ) (see Table 1). A spectral analysis of the detected excess events shows that the differential energy spectrum of photons is consistent with a simple power law $\mathrm{d} N / \mathrm{d} E=N_{0}(E / 1 \mathrm{TeV})^{-\Gamma}$ with a flux normalisation at $1 \mathrm{TeV}$ of $N_{0}=\left(1.95 \pm 0.32_{\text {stat }} \pm 0.39_{\text {syst }}\right) \times$ $10^{-12} \mathrm{TeV}^{-1} \mathrm{~cm}^{-2} \mathrm{~s}^{-1}$ and a spectral index $\Gamma=2.92 \pm 0.30_{\text {stat }} \pm$ $0.20_{\text {syst }}$ (see Fig. 1 and Table 1 ) with a fit probability of 0.64 . The integral flux above $1 \mathrm{TeV}$ averaged over the whole observation period is $F(E>1 \mathrm{TeV})=\left(1.01 \pm 0.18_{\text {stat }} \pm 0.20_{\text {sys }}\right) \times$ $10^{-12} \mathrm{~cm}^{-2} \mathrm{~s}^{-1}$.

\footnotetext{
1 Paris Analysis software version 0-8-18.

2 H.E.S.S. Analysis Package (HAP) version 11-02-pl04.
} 


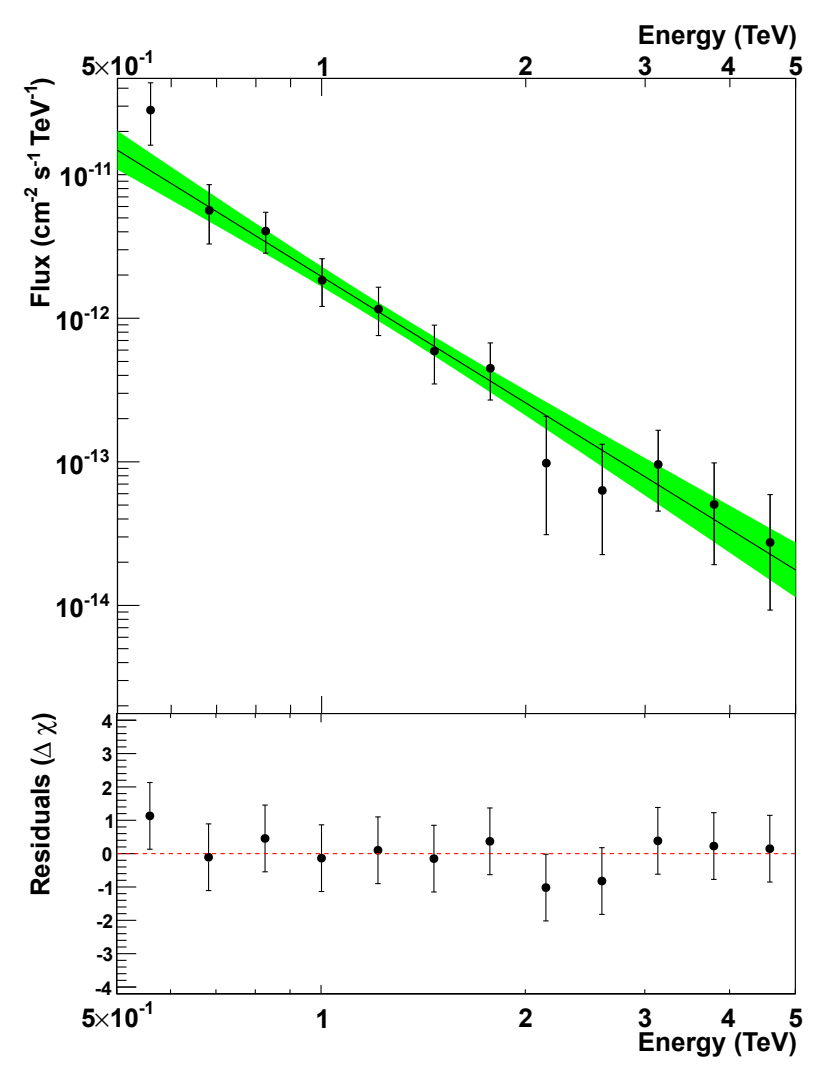

Fig. 1. Overall differential energy spectrum of the VHE $\gamma$-ray emission from PSR B1259-63/LS 2883 for the whole observation period from 9th to 16th of January 2011. The solid line denotes the spectral fit with a simple power law. The green band represents the $1 \sigma$ confidence interval. Points are derived for the minimum significance of $1.5 \sigma$ per bin. Points' error bars represent $1 \sigma$ errors.

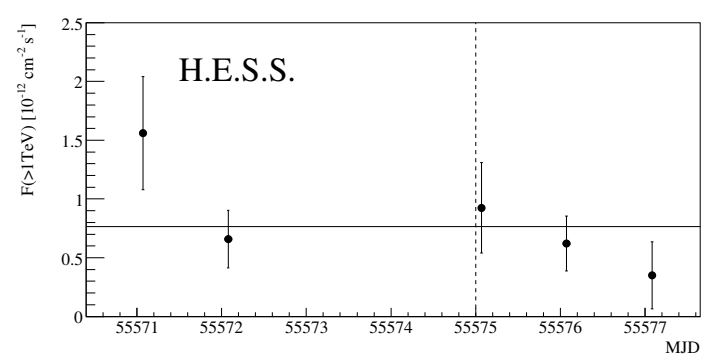

Fig. 2. Integrated photon flux above $1 \mathrm{TeV}$ for individual observation nights. The solid horizontal line indicates the fit of a constant to the distribution. The flare start date is indicated by the dashed vertical line.

\subsection{Light curves}

To check for variability of the source a light curve was produced on a night-by-night basis assuming the photon spectral index obtained in the spectral fit (Fig. 2). The spectral index was fixed at the value obtained in the spectral analysis of the total data because of the low statistics for each individual night. The light curve is consistent with a constant resulting in a mean flux of $(0.77 \pm 0.13) \times 10^{-12} \mathrm{~cm}^{-2} \mathrm{~s}^{-1}$ (horizontal line in Fig. 2) with $\chi^{2} / \mathrm{NDF}=6.35 / 4$ (corresponds to the probability of 0.17 ; NDF is the number of degrees of freedom), yielding no evidence for variability in the seven-nights observation period. For each individual night the source is detected at a statistical significance level $>3 \sigma$ except for the last point, whose significance is only $1.5 \sigma$.

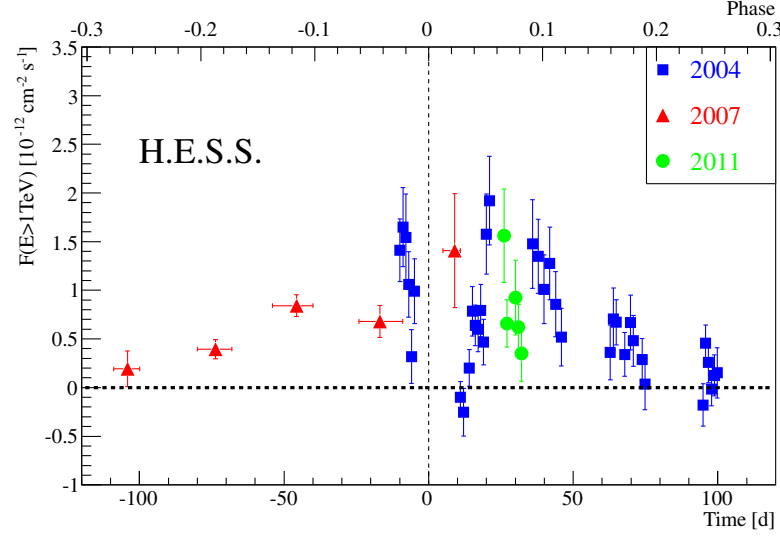

Fig. 3. Integrated photon flux above $1 \mathrm{TeV}$ as a function of the time with respect to the periastron passage indicated with the vertical dashed line. The corresponding orbital phases (mean anomaly) are shown on the upper horizontal axis. The data from the 2004 (blue squares) (Aharonian et al. 2005b), 2007 (red triangles) (Aharonian et al. 2009), and 2011 (green circles) observation campaigns are shown. For the 2004 and 2011 data the flux is shown in daily bins while for the 2007 data the flux is shown in monthly bins for clarity.

For a comparison with the GeV flare (see Sect. 4.2) the whole dataset was divided into two datasets: "pre-flare" $\left(t_{\mathrm{p}}+26 \mathrm{~d}\right.$ to $\left.t_{\mathrm{p}}+29 \mathrm{~d}\right)$ and "flare" $\left(t_{\mathrm{p}}+30 \mathrm{~d}\right.$ to $\left.t_{\mathrm{p}}+32 \mathrm{~d}\right)$. These two datasets were analysed independently and revealed similar fluxes and significance levels (see Table 1). A spectral analysis of the two datasets shows that both spectra are consistent with a simple power law, yielding similar values of the spectral index (see Table 1). The two spectral indices are consistent with the one obtained for the total dataset. These results are discussed in Sect. 4.2.

\subsection{Re-analysis of the 2004 data}

For the data taken around the 2004 periastron passage the energy spectrum had been measured only up to $\sim 3 \mathrm{TeV}$ (Aharonian et al. 2005b) while for the much smaller dataset of 2011 observations the spectrum was measured up to $>4 \mathrm{TeV}$ and for the comparable dataset of 2007 observations up to $>10 \mathrm{TeV}$ (Aharonian et al. 2009) using more advanced analysis techniques with a better understanding of weak fluxes. To fully compare observation results around different periastron passages (see below), the 2004 data were re-analysed with the current analysis techniques, the same as used for the analysis of the 2011 data described above. The re-analysis results are compatible with the published ones. The differential energy spectrum measured up to $\sim 10 \mathrm{TeV}$ is consistent with a power law with a spectral in$\operatorname{dex} \Gamma=2.8 \pm 0.1_{\text {stat }} \pm 0.2_{\text {syst }}$ and a flux normalisation at $1 \mathrm{TeV}$ of $N_{0}=\left(1.29 \pm 0.08_{\text {stat }} \pm 0.26_{\text {syst }}\right) \times 10^{-12} \mathrm{TeV}^{-1} \mathrm{~cm}^{-2} \mathrm{~s}^{-1}$. The new analysis of the 2004 data is therefore compatible with the published results when extrapolated above $3 \mathrm{TeV}$.

\section{Discussion}

\subsection{Comparison with previous H.E.S.S. observations}

In Fig. 3, the integrated photon flux above $1 \mathrm{TeV}$ as a function of time with respect to periastron (indicated by the dashed vertical line) is shown. The light curve compiles the data from all three periastron observation campaigns spanning from 100 days before to 100 days after the periastron. The observed flux from 
H.E.S.S. collaboration: H.E.S.S. Observations of PSR B1259-63/LS 2883 around the 2010/2011 periastron passage

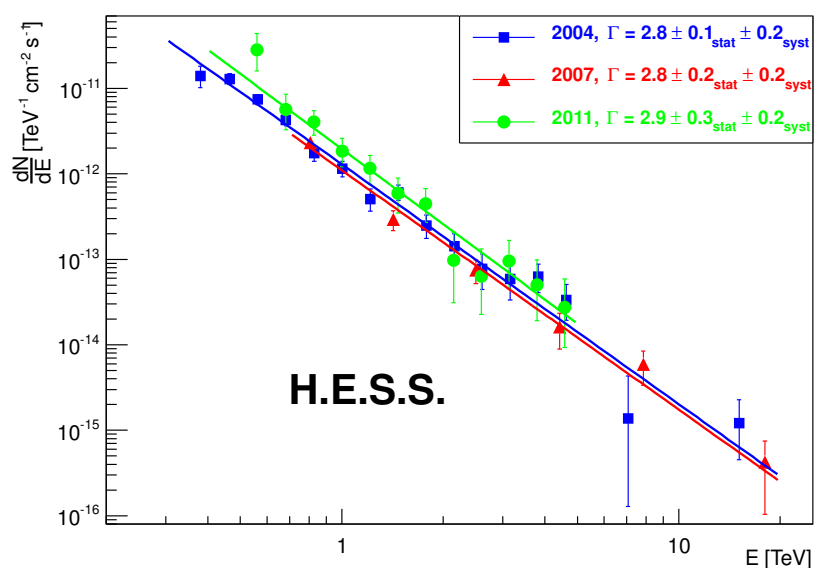

Fig. 4. Differential energy spectra of the VHE $\gamma$-ray emission from PSR B1259-63/LS 2883 for the data collected around the 2004 (blue squares), 2007 (red triangles), and 2010/2011 (green circles) periastron passages. For the 2004 data the spectrum presented in this paper is shown. The 2007 spectrum is extracted from Aharonian et al. (2009).

the 2010/2011 observation campaign is compatible with the flux detected in 2004 at the similar orbital phases. Observation periods from 2004 and 2007 were separated in time with respect to the periastron position, i.e., observations in 2004 were performed mainly after and in 2007 mainly before the periastron. Therefore, it was impossible to directly confirm the repetitive behaviour of the source by comparing observations of PSR B1259-63/LS 2883 at the same orbital phases. In this perspective, although the 2011 observations do not exactly overlap with the orbital phases of previous studies, they cover the gap in the 2004 data post-periastron light curve and the integrated flux follows the shape of the light curve, yielding a stronger evidence for the repetitive behaviour of the source.

The spectral shape of the VHE $\gamma$-ray emission from PSR B1259-63/LS 2883 around the 2010/2011 periastron passage is similar to what was observed during previous periastron passages (Fig. 4). The photon index of $2.92 \pm 0.25_{\text {stat }} \pm 0.2_{\text {syst }}$ inferred from the 2011 data is well compatible with previous results. The spectrum measured for the 2011 data can be resolved only up to $\sim 4 \mathrm{TeV}$, which is explained by a very low statistics at higher energies due to a short exposure of the source.

\subsection{Search for the equivalent "GeV Flare" in the H.E.S.S. data}

The absence of the flux enhancement during the $\mathrm{GeV}$ flare at radio and $\mathrm{X}$-ray wavebands indicates that the $\mathrm{GeV}$ flare may be created by physical processes different from the those responisble for the emission at other wavelengths. The VHE postperiastron data obtained with H.E.S.S. around the 2004 periastron passage do not show any evidence of a flux outburst at orbital phases at which the $\mathrm{GeV}$ flare is observed. However, the H.E.S.S. observations around the 2004 periastron passage do not comprise the orbital phase when the $\mathrm{GeV}$ flare starts. Moreover, to compare H.E.S.S. 2004 data with the GeV flare observed after the 2010 periastron passage, one has to assume that the $\mathrm{GeV}$ flare is a periodic phenomenon, which may not be the case. The H.E.S.S. data taken between 9 th and 16th of January in 2011 provide a three-day overlap in time with the $\mathrm{GeV}$ flare. Therefore, it is possible to directly study any flux enhancement in the VHE band on the time scale of the HE flare. To improve the sensitivity of the variability search the whole period of the
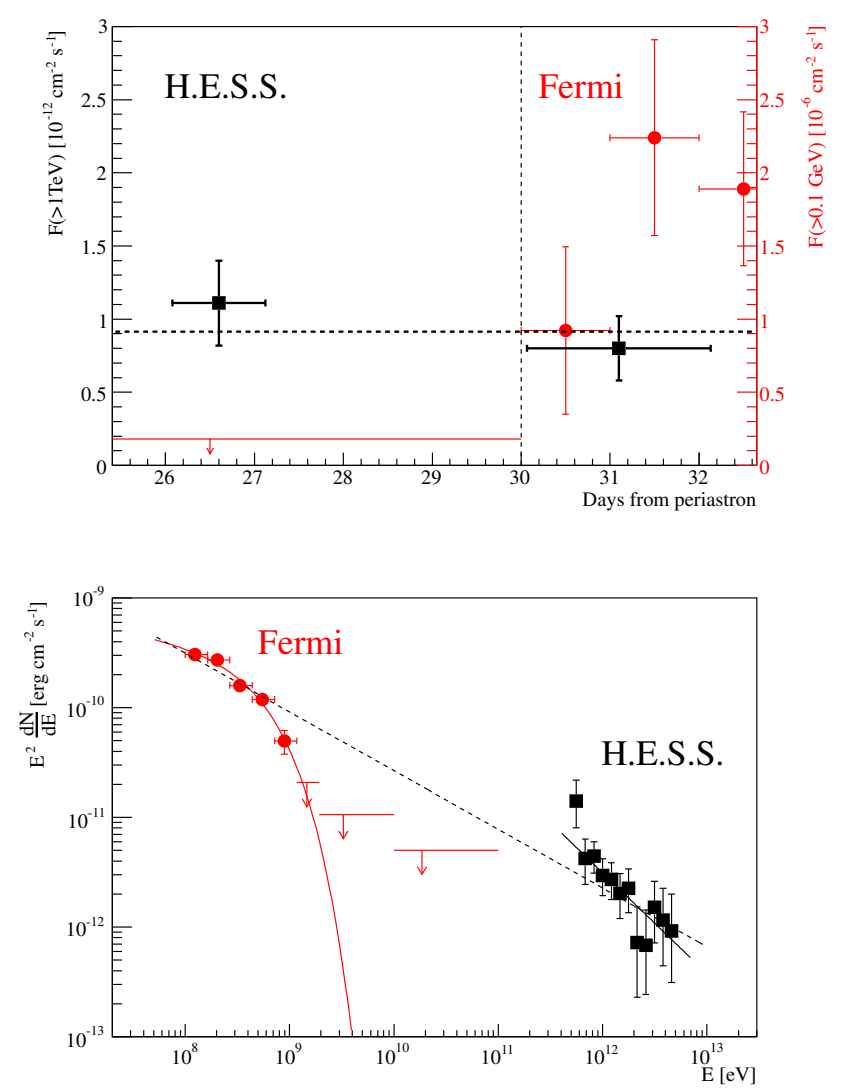

Fig. 5. (Top) Integrated photon fluxes above $1 \mathrm{TeV}$ for the pre-flare and flare periods (see text) are shown as black filled boxes. The dashed horizontal line shows the best fit with a constant. The HE data points above $0.1 \mathrm{GeV}$ as reported by Abdo et al. (2011) are shown as red filled circles. The flare start date is indicated by the dashed vertical line. The left axis indicates the units for the VHE flux and the right (red) axis denotes the units for the HE flux. (Bottom) The spectral energy distribution of the HE-VHE emission. For the HE emission the overall flare spectrum is shown as reported by Abdo et al. (2011). Marking of the data points is the same as in the top panel. Solid lines denote the fit of the Fermi data only with the power law with exponential cut-off (red) and the fit of the H.E.S.S. data only with the power law (black). The dashed black line denotes the fit of the Fermi (excluding upper limits) and H.E.S.S. data together with the power law.

H.E.S.S observations was divided into two time periods of almost equal length: before ("pre-flare") and during ("flare") the HE flare (see Sect. 3.4). The pre-flare and flare dataset analysis results are presented in Table 1.

To search for variability, the flux as a function of time was fitted with a constant, which resulted in a mean flux of $(0.91 \pm 0.18) \times 10^{-12} \mathrm{~cm}^{-2} \mathrm{~s}^{-1}$ (black horizontal dashed line in Fig. 5 top). The fit has a $\chi^{2}$-to-NDF ratio of $0.73 / 1$, which corresponds to a $\chi^{2}$ probability of 0.39 , showing no indication for a flux enhancement. Note that the spectral parameters obtained by an independent fit of each of the two periods have been used here.

If one assumes that HE and VHE emission are created according to the same scenario, i.e. the same acceleration and radiation processes and sites, then a flux enhancement of the same magnitude as observed at HEs should be also seen at VHEs. To investigate this hypothesis, the flare coefficient $\kappa$ is introduced as the ratio of the fluxes during the flare period and the pre-flare period. The ratio of the HE $(E>0.1 \mathrm{GeV})$ flux averaged over the three-day interval between $\left(t_{\mathrm{p}}+30 \mathrm{~d}\right)$ and $\left(t_{\mathrm{p}}+32 \mathrm{~d}\right)$ to the upper limit on the HE pre-flare emission (see Fig. 5 top) yields 
a lower limit on the HE emission flare coefficient $\kappa_{\mathrm{HE}} \geq 9.2$. An upper limit on the VHE flare coefficient can be estimated using the profile likelihood method. The likelihood function is defined as a product of two Gaussian distributions of the pre-flare and flare flux measurements $\phi_{1}$ and $\phi_{2}$ correspondingly, stating that the flare measurement $\phi_{2}$ varies around $\tilde{\kappa} \tilde{\phi_{1}}$, where the tilde denotes the true value for a parameter. The profile likelihood $\lambda$ is then built as a function of $\kappa$ :

$\lambda(\kappa)=\frac{L\left(\hat{\phi}_{1}, \kappa \mid \phi_{1}, \phi_{2}\right)}{L\left(\hat{\phi}_{1}, \hat{\kappa} \mid \phi_{1}, \phi_{2}\right)}$.

Where the hat denotes the maximum likelihood estimate for a parameter. The variable $-2 \log \lambda$ follows a $\chi^{2}$ distribution with one degree of freedom, which allows one to calculate the $99.7 \%$ confidence level (equivalent of $3 \sigma$ ) upper limit of $\kappa_{99.7 \%}<3.5$. The obtained upper limit is lower than the observed lower limit on $\kappa_{\mathrm{HE}}$.

The statistical tests presented above give two main results:

- A flare of similar magnitude as observed in the HE band can be firmly rejected in the VHE band at the same orbital phase.

- There is no significant difference between the pre-flare and the flare flux in the VHE band.

These two results suggest that the HE flare emission has a different nature than the VHE emission.

This conclusion is also supported by the inconsistency of HE and VHE emission spectra (see Fig. 5 bottom). The joint fit of the Fermi and H.E.S.S. data points with the simple power law (the dashed line on Fig. 5 bottom) results in a fit probability of 0.004 and, hence, fails to explain the combined HE/VHE emission even ignoring the Fermi upper limits, which cannot be taken into account in the fit procedure. Moreover, the Fermi upper limits at $1-100 \mathrm{GeV}$ violate any reasonable model that would be able to explain the HE and VHE emission together. The Fermi spectrum alone is consistent with the the power law with exponential cut-off $E^{2} \mathrm{~d} N / \mathrm{d} E=N_{0}(E / 0.1 \mathrm{GeV})^{-p} e^{-E / E_{\text {cutoff }}}$ with the index $p=0.16 \pm 0.32$, the cut-off energy of $E_{\text {cutoff }}=0.5 \pm 0.2 \mathrm{GeV}$ and the normalisation $N_{0}=(4 \pm 0.4) \times 10^{-10} \mathrm{erg} \mathrm{cm}^{-2} \mathrm{~s}^{-1}$. The fit probability is 0.27 .

Several models have been proposed to explain the VHE emission from the source. In a hadronic scenario, the VHE $\gamma$-ray emission could be produced by the interaction of the ultrarelativistic pulsar wind particles with the dense equatorial disk outflow with subsequent production of $\pi^{0}$ pions and hence VHE $\gamma$-rays (Kawachi et al. 2004; Neronov \& Chernyakova 2007). However, the detection of the source before the expected disk passage in 2007 casts doubts on the hadronic scenario, suggesting that the VHE emission should be created at least partly by leptonic processes (Aharonian et al. 2009). Within the leptonic scenario, VHE emission from PSR B1259-63/LS 2883 is explained by the inverse Compton (IC) scattering of shockaccelerated electrons on stellar photons (Tavani \& Arons 1997; Kirk et al. 1999; Dubus 2006; Khangulyan et al. 2007).

Few possible explanations for the nature of the HE flare are discussed in the literature. One of them, suggested by Khangulyan et al. (2012), is based on IC scattering of the unshocked pulsar wind on the stellar and circumstellar disk photons. While the pulsar is inside the disk, the IC scattering of the unshocked pulsar wind is supressed due to the high ram pressure. But immediately after the pulsar escapes the disk in the postperiastron phase, the unshocked pulsar wind zone towards the observer increases significantly, while the density of the circumstellar disk photons is still high enough for efficient IC scattering. Therefore, the enhancement of the HE flux is observed. This is not expected in the pre-periastron phase because the termination shock should expand towards the direction opposite to the observer. This model also predicts the difference between the HE and VHE emission, the latter expected to result from the upscattering of the stellar photons by the electrons accelerated at the termination shock between pulsar and stellar winds. Another explanation of the HE flare can be the Doppler boosting of the radiation created by the shocked pulsar wind (Bogovalov et al. 2008; Dubus et al. 2010; Kong et al. 2012). It is unclear though why the flare is not detected at other wavebands, since the Doppler boosting should also enhance X-ray and VHE $\gamma$-ray fluxes. This question, however, can be resolved assuming a specific anisotropy of the pulsar wind and the difference of the emission behaviour in different regions of the termination shock, the isotropic emission in the apex and the beamed emission in the tail of the shock (Kong et al. 2012). In this particular case the HE flare is explained by the Doppler boosting of the synchrotron emission. Abdo et al. (2011) suggested that the flare can also be explained by an anisotropy of the pulsar wind and/or stellar material. The anisotropy of electrons with the highest energies would cause an anisotropy of the synchrotron radiation at high energies. In this interpretation, the HE emission is produced by the synchrotron mechanism. The local increase of the stellar wind density would increase the Bremsstrahlung component, which may also cause the HE flare. Regardless of, which mechanism is responsible for the HE flare, the fact that it is observed only after the periastron indicates either a strong dependency of the HE emission on the geometry of the system, i.e., its configuration with respect to the direction to the observer, or some local perturbation of the stellar material. A detailed interpretation of the HE-VHE emission is beyond the scope of this paper and will be discussed in the joint MWL paper.

\section{Summary}

The binary system PSR B1259-63/LS 2883 was monitored by H.E.S.S. around the periastron passage on 15 th of December 2010. The observed flux and spectral shape agree well with what was measured during previous periastron passages. The observations were performed at similar orbital phases as around the 2004 periastron passage, strengthening the evidence for the repetitive behaviour of the source at VHEs.

H.E.S.S. observations were part of a joint MWL campaign that also included radio, optical, X-ray, and HE observations. A spectacular flare observed at HEs with Fermi LAT overlapped in time with the H.E.S.S. observations. A careful statistical study showed that the HE flare does not have a counterpart at VHEs, indicating that the HE and VHE emissions are produced in different physical scenarios.

Acknowledgements. The support of the Namibian authorities and of the University of Namibia in facilitating the construction and operation of H.E.S.S. is gratefully acknowledged, as is the support by the German Ministry for Education and Research (BMBF), the Max Planck Society, the French Ministry for Research, the CNRS-IN2P3 and the Astroparticle Interdisciplinary Programme of the CNRS, the UK Particle Physics and Astronomy Research Council (PPARC), the IPNP of the Charles University, the South African Department of Science and Technology and National Research Foundation, and by the University of Namibia. We appreciate the excellent work of the technical support staff in Berlin, Durham, Hamburg, Heidelberg, Palaiseau, Paris, Saclay, and in Namibia in the construction and operation of the equipment.

\section{References}

Abdo, A. A., Ackermann, M., Ajello, M., et al. 2010, ApJS, 188, 405 Abdo, A. A., Ackermann, M., Ajello, M., et al. 2011, ApJ, 736, L11 
Abramowski, A, et al. (H.E.S.S. Collaboration) 2012, A\&A, 541, A5

Aharonian, F., Akhperjanian, A. G., Aye, K.-M., et al. 2005a, Science, 309, 746 Aharonian, F., Akhperjanian, A. G., Aye, K.-M., et al. 2005b, A\&A, 442, 1

Aharonian, F., Akhperjanian, A. G., Bazer-Bachi, A. R., et al. 2006, A\&A, 457, 899

Aharonian, F. A., Akhperjanian, A. G., Bazer-Bachi, A. R., et al. 2007, A\&A, 469, L1

Aharonian, F., Akhperjanian, A. G., Anton, G., et al. 2009, A\&A, 507, 389

Albert, J., Aliu, E., Anderhub, H., et al. 2006, Science, 312, 1771

Albert, J., Aliu, E., Anderhub, H., et al. 2007, ApJ, 665, L51

Bogovalov, S. V., Khangulyan, D. V., Koldoba, A. V., Ustyugova, G. V., \& Aharonian, F. A. 2008, MNRAS, 387, 63

Chernyakova, M., Neronov, A., Lutovinov, A., Rodriguez, J., \& Johnston, S. 2006, MNRAS, 367, 1201

Chernyakova, M., Neronov, A., Aharonian, F., Uchiyama, Y., \& Takahashi, T. 2009, MNRAS, 397, 2123

Cominsky, L., Roberts, M., \& Johnston, S. 1994, ApJ, 427, 978

Connors, T. W., Johnston, S., Manchester, R. N., \& McConnell, D. 2002, MNRAS, 336, 1201

de Naurois, M., \& Rolland, L. 2009, Astropart. Phys., 32, 231

Dubus, G. 2006, A\&A, 456, 801

Dubus, G., Cerutti, B., \& Henri, G. 2010, A\&A, 516, A18

Hinton, J. A. 2004, New Astron. Rev., 48, 331

Hirayama, M., Cominsky, L. R., Kaspi, V. M., et al. 1999, ApJ, 521, 718

Johnston, S., Lyne, A. G., Manchester, R. N., et al. 1992a, MNRAS, 255, 401

Johnston, S., Manchester, R. N., Lyne, A. G., et al. 1992b, ApJ, 387, L37

Johnston, S., Manchester, R. N., McConnell, D., \& Campbell-Wilson, D. 1999, MNRAS, 302, 277

Johnston, S., Ball, L., Wang, N., \& Manchester, R. N. 2005, MNRAS, 358, 1069

Kaspi, V. M., Tavani, M., Nagase, F., et al. 1995, ApJ, 453, 424

Kawachi, A., Naito, T., Patterson, J. R., et al. 2004, ApJ, 607, 949

Kerschhaggl, M. 2011, A\&A, 525, A80

Khangulyan, D., Hnatic, S., Aharonian, F., \& Bogovalov, S. 2007, MNRAS, 380, 320

Khangulyan, D., Aharonian, F. A., Bogovalov, S. V., \& Ribó, M. 2012, ApJ, 752, L17

Kirk, J. G., Ball, L., \& Skjaeraasen, O. 1999, Astropart. Phys., 10, 31

Kong, S. W., Cheng, K. S., \& Huang, Y. F. 2012, ApJ, 753, 127

Li, T.-P., \& Ma, Y.-Q. 1983, ApJ, 272, 317

Melatos, A., Johnston, S., \& Melrose, D. B. 1995, MNRAS, 275, 381

Moldón, J., Johnston, S., Ribó, M., Paredes, J. M., \& Deller, A. T. 2011, ApJ, 732, L10

Negueruela, I., Ribó, M., Herrero, A., et al. 2011, ApJ, 732, L11

Neronov, A., \& Chernyakova, M. 2007, Ap\&SS, 309, 253

Shaw, S. E., Chernyakova, M., Rodriguez, J., et al. 2004, A\&A, 426, L33

Tam, P. H. T., Huang, R. H. H., Takata, J., et al. 2011, ApJ, 736, L10

Tavani, M., \& Arons, J. 1997, ApJ, 477, 439

Wang, N., Johnston, S., \& Manchester, R. N. 2004, MNRAS, 351, 599

1 Universität Hamburg, Institut für Experimentalphysik, Luruper Chaussee 149, 22761 Hamburg, Germany

2 Laboratoire Univers et Particules de Montpellier, Université Montpellier 2, CNRS/IN2P3, CC 72, Place Eugène Bataillon, 34095 Montpellier Cedex 5, France

3 Max-Planck-Institut für Kernphysik, PO Box 103980, 69029 Heidelberg, Germany

4 Dublin Institute for Advanced Studies, 31 Fitzwilliam Place, Dublin 2, Ireland

5 National Academy of Sciences of the Republic of Armenia, Yerevan, Armenia

6 Yerevan Physics Institute, 2 Alikhanian Brothers St., 375036 Yerevan, Armenia

7 Universität Erlangen-Nürnberg, Physikalisches Institut, ErwinRommel-Str. 1, 91058 Erlangen, Germany

8 University of Durham, Department of Physics, South Road, Durham DH1 3LE, UK
9 DESY, 15735 Zeuthen, Germany

10 Institut für Physik und Astronomie, Universität Potsdam, KarlLiebknecht-Strasse 24/25, 14476 Potsdam, Germany

11 Nicolaus Copernicus Astronomical Center, ul. Bartycka 18, 00-716 Warsaw, Poland

12 CEA Saclay, DSM/Irfu, 91191 Gif-Sur-Yvette Cedex, France

13 Landessternwarte, Universität Heidelberg, Königstuhl, 69117 Heidelberg, Germany

14 APC, AstroParticule et Cosmologie, Université Paris Diderot, CNRS/IN2P3, CEA/Irfu, Observatoire de Paris, Sorbonne Paris Cité, 10 rue Alice Domon et Léonie Duquet, 75205 Paris Cedex 13, France

15 Laboratoire Leprince-Ringuet, École Polytechnique, CNRS/IN2P3, 91128 Palaiseau, France

16 Institut für Theoretische Physik, Lehrstuhl IV: Weltraum und Astrophysik, Ruhr-Universität Bochum, 44780 Bochum, Germany

17 Institut für Physik, Humboldt-Universität zu Berlin, Newtonstr. 15, 12489 Berlin, Germany

18 LUTH, Observatoire de Paris, CNRS, Université Paris Diderot, 5 place Jules Janssen, 92190 Meudon, France

19 LPNHE, Université Pierre et Marie Curie Paris 6, Université Denis Diderot Paris 7, CNRS/IN2P3, 4 place Jussieu, 75252 Paris Cedex 5, France

20 Institut für Astronomie und Astrophysik, Universität Tübingen, Sand 1, 72076 Tübingen, Germany

21 Astronomical Observatory, The University of Warsaw, Al. Ujazdowskie 4, 00-478 Warsaw, Poland

22 Unit for Space Physics, North-West University, Potchefstroom 2520, South Africa

23 School of Physics, University of the Witwatersrand, 1 Jan Smuts Avenue, Braamfontein, 2050 Johannesburg, South Africa

24 Oskar Klein Centre, Department of Physics, Stockholm University, Albanova University Center, 10691 Stockholm, Sweden

25 Université Bordeaux 1, CNRS/IN2P3, Centre d'Études Nucléaires de Bordeaux Gradignan, 33175 Gradignan, France

26 Funded by contract ERC-StG-259391 from the European Community

27 University of Namibia, Department of Physics, Private Bag 13301, Windhoek, Namibia

28 School of Chemistry \& Physics, University of Adelaide, 5005 Adelaide, Australia

29 UJF-Grenoble 1/CNRS-INSU, Institut de Planétologie et d'Astrophysique de Grenoble (IPAG) UMR 5274, 38041 Grenoble, France

30 Department of Physics and Astronomy, The University of Leicester, University Road, Leicester, LE1 7RH, UK

31 Instytut Fizyki Jạdrowej PAN, ul. Radzikowskiego 152, 31-342 Kraków, Poland

32 Institut für Astro- und Teilchenphysik, Leopold-FranzensUniversität Innsbruck, 6020 Innsbruck, Austria

33 Laboratoire d'Annecy-le-Vieux de Physique des Particules, Université de Savoie, CNRS/IN2P3, 74941 Annecy-le-Vieux, France

34 Obserwatorium Astronomiczne, Uniwersytet Jagielloński, ul. Orla 171, 30-244 Kraków, Poland

35 Toruń Centre for Astronomy, Nicolaus Copernicus University, ul. Gagarina 11, 87-100 Toruń, Poland

36 Charles University, Faculty of Mathematics and Physics, Institute of Particle and Nuclear Physics, V Holešovičkách 2, 18000 Prague 8, Czech Republic

37 School of Physics \& Astronomy, University of Leeds, Leeds LS2 9JT, UK 